\title{
Nonlinear interaction of charged particles with strong laser pulses in a gaseous media
}

\author{
H. K. Avetissian, ${ }^{*}$ S. S. Israelyan, and Kh. V. Sedrakian \\ Department of Quantum Electronics, Yerevan State University, 1 A. Manukian, Yerevan 375025, Armenia
}

(Received 23 May 2007; published 11 July 2007)

\begin{abstract}
The charged particles nonlinear dynamics in the field of a strong electromagnetic wave pulse of finite duration and certain form of the envelope, in the refractive medium with a constant and variable refraction indexes, is investigated by means of numerical integration of the classical relativistic equations of motion. The particle energy dependence on the pulse intensity manifests the nonlinear threshold phenomenon of a particle reflection and capture by actual laser pulses in dielectric-gaseous media that takes place for a plane electromagnetic wave in the induced Cherenkov process. Laser acceleration of the particles in the result of the reflection from the pulse envelope and in the capture regime with the variable refraction index along the pulse propagation direction is investigated.
\end{abstract}

DOI: 10.1103/PhysRevSTAB.10.071301

PACS numbers: 41.75.Jv, 41.75.Ht, 41.60.Bq

\section{INTRODUCTION}

The problem of the creation of new types of ultrahigh energy accelerators of the charged particles, in particular, laser and plasma accelerators of the small sizes, is one of the most important problems in the modern physics of high energy and elementary particles, accelerators, plasmas, and lasers physics [1-3]. The recent achievements in the laser technology related to implementation of supershortfemtosecond laser sources and subcycle pulses of relativistic intensities exceeding the intra-atomic fields open real opportunities for the realization of laser-plasma accelerators of ultrahigh energies [4-10]. These schemes are based on the laser-plasma-wakefield and laser-beat wave mechanisms of laser-assisted acceleration in plasma. The interaction of such powerful radiation fields with the plasma has led to the generation of quasimonoenergetic relativistic electron beams in laser-driven plasma accelerators [1117]. Concerning the interaction of ultrashort laser pulses of relativistic intensities with the free electrons in vacuum, i.e., the implementation of laser accelerators, one should note that experiments in this area are gathering power at present [18-24].

One of the first laser accelerator concepts is the inverse Cherenkov accelerator (ICA) scheme that makes a phase velocity matching of electromagnetic (e.m.) wave with a particle by controlling the refractive index of gaseous medium so as to be a wave phase velocity in a dielectric medium less than light speed in vacuum. The ICA has been well investigated theoretically and experimentally since the advent of lasers (see, for example, [25-28]).

The exact solution of relativistic equations of motion (both classical and quantum) of a charged particle in the field of a plane wave pulse in a medium (at the neglect of the dispersion for a quasimonochromatic wave pulse) $[29,30]$ shows that the induced Cherenkov process has a threshold over the wave intensity-critical value-at

*avetissian@ysu.am which the nonlinear resonance with the stimulated wave proceeds. If a wave intensity exceeds this critical value, a transverse wave becomes a potential barrier for the external particle, and a potential well for an internal one, and the particles "reflection" or capture by a plane e.m. wave occurs, respectively [29-31]. After the reflection, the particle acceleration or deceleration (wave amplification) takes place depending on the initial conditions (the wave overtakes the particle or vice versa). As to the phenomenon of capture [31], the longitudinal velocity of the particle in this case oscillates in time remaining at the average constant. However, it is also possible to accelerate a particle in the capture regime if one changes (decreases) the refraction index of the gaseous medium by self-consistent law, continuously increasing the phase velocity of the wave [32].

The nonlinear threshold phenomena of particle reflection or capture by a plane e.m. wave take place in the induced Compton [33] and undulator processes [34] as well, i.e., it is the feature of coherent radiation processes at which the effective interaction occurs with a slowed traveling wave propagating with the phase velocity less than light speed in vacuum. The existence of a critical field essentially changes the character of the particle-plane wave interaction in the mentioned processes. The critical intensity determining the threshold of the process is a significant parameter at the particle-wave interaction in coherent radiation processes, where at the above critical value of intensity the particle does not experience a periodic wave field/photons. For example, at the above critical intensities, one cannot speak about Compton effect in dielectric media (it takes place only at the below-threshold interaction) [35]. It is important to note that the effect of the critical field exists even in a very weak wave field if the interaction occurs in the region close to the initial Cherenkov resonance. Therefore one cannot escape these peculiarities restricting only the intensity of the external wave field and solve the problem in the scope of the linear theory even for very weak wave fields [36]. 
Over 25 years later and without quotation on the original works, the particle reflection phenomenon from a slowed plane wave pulse has been applied also for cases where the group velocity of a wave pulse due to the dispersion can be less than light speed in vacuum [37-39]: in Ref. [37]—for the case of a plasma' in Refs. [38,39] — at the focusing of an ultrashort laser pulse in vacuum, in the so-called by authors "dispersion-dominated propagation regime." In Ref. [37] any consideration of a medium dispersion is not made, meanwhile the group velocity arises for a wave packet and is different from the phase velocity in a medium, namely, because of dispersion. So, the vector potential in Ref. [37] is written formally without the definition of electric and magnetic fields for a wave pulse in a dispersive medium according to Maxwell equations. At the physical consideration of the dispersion, the authors should find from the Maxwell equations an exact relation between the electric and magnetic fields for a wave pulse in a dispersive medium then introduce the corresponding vector potential. Meanwhile, via an indefinite vector potential of a wave pulse in plasma with the dispersion, they repeat the exact results for the particle reflection phenomenon, only instead of the slowed phase velocity they have a slowed group velocity. However, even in such a consideration (and apart from that the analytic solution in Ref. [37] corresponds only to a particular case if initially particle has not a transversal velocity and a wave pulse has a circular polarization), the used integral of motion is not exact. The latter is adiabatic invariant corresponding to the assumed form of the vector potential (see the amplitude and phase of a wave with its arguments in Ref. [37]) and initial conditions of adiabatic interaction. Namely, only at the adiabatic variation of the wave amplitude can one introduce the assumed group velocity. Hence, the authors cannot ignore this fact and apply these solutions (the same concerns the papers $[38,39]$ as well) for ultrashort laser pulses providing current superintensities. Nevertheless, the efficient laserdriven acceleration schemes based on the particle reflection phenomenon due to the slowed group velocity of a high-intensity laser pulse in plasma [37] and from the leading front of an supershort laser pulse of ultrarelativistic intensities in vacuum $[38,39]$ have been suggested.

The particle reflection phenomenon has a significant property: at the above-threshold interaction the induced process occurs only in one direction - either direct (laser scheme) [31] or inverse (accelerator scheme) [32]; meanwhile at the below-threshold interaction both direct and inverse processes take place simultaneously which reduces in general the power gain for each problem. Therefore this phenomenon gives in principal new opportunity for free electron lasers or laser accelerators.

The goal of this paper is to clear up the influence of finite duration and transverse width of actual laser pulses on the reflection or capture phenomena. The exact solution of relativistic equations of motion which revealed these phe- nomena has been obtained for a plane e.m. wave (capture phenomenon-for a monochromatic wave) [29,30,32]. For a wave pulse of finite duration and transverse width, such as actual laser pulses are, it is impossible to solve the problem analytically. Hence, in the current paper we will investigate the reflection and capture phenomena in case of actual laser pulses by numerical integration of relativistic equations of motion. Since the result of reflection corresponds to particle acceleration or deceleration depended on initial conditions, we will present the results for acceleration. The capture phenomenon can lead to particle acceleration if one decreases continuously the refractive index of a gaseous medium. So, to prove the capture phenomenon (the existence of the critical point) by actual laser pulses, we will consider the acceleration problem in a gaseous medium with a variable refractive index.

The organization of the paper is as follows. In Sec. II we treat the nonlinear dynamics of a charged particle in the field of strong laser pulse in gaseous medium with a constant refraction index. In Sec. III we present the simulations for particle nonlinear dynamics with subsequent acceleration in gaseous medium, the refraction index of which continuously decreases along the wave propagation direction. Finally, conclusions are given in Sec. IV.

\section{NONLINEAR DYNAMICS OF A CHARGED PARTICLE IN THE FIELD OF STRONG LASER PULSE IN A MEDIUM}

For numerical investigation of the entire dynamics of a charged particle and its acceleration by actual laser pulses, it is convenient to represent the equations of motion of a particle in the form of dimensionless quantities:

$$
\begin{gathered}
\frac{d \Pi}{d t^{\prime}}=\xi_{E}\left(t^{\prime}, \mathbf{r}^{\prime}\right)+\frac{1}{\sqrt{1+\Pi^{2}}}\left[\Pi \times \xi_{H}\left(t^{\prime}, \mathbf{r}^{\prime}\right)\right], \\
\frac{d \mathbf{r}^{\prime}}{d t^{\prime}}=\frac{\Pi}{\sqrt{1+\Pi^{2}}},
\end{gathered}
$$

where the dimensionless momentum $\Pi$, time $t^{\prime}$, and coordinate $\mathbf{r}^{\prime}$ are introduced:

$$
\Pi=\frac{\mathbf{p}}{m c}, \quad t^{\prime}=\omega t, \quad \mathbf{r}^{\prime}=\frac{\omega}{c} \mathbf{r},
$$

$\omega$ is the carrier frequency of a laser pulse, and the dimensionless parameters

$$
\xi_{E}=\frac{e \mathbf{E}}{m c \omega}, \quad \xi_{H}=\frac{e \mathbf{H}}{m c \omega}
$$

characterize the electric (E) and magnetic (H) field strengths of the wave pulse.

The particle energy change is given by the equation

$$
\frac{d \gamma}{d t^{\prime}}=\frac{\Pi \xi_{E}\left(t^{\prime}, \mathbf{r}^{\prime}\right)}{\sqrt{1+\Pi^{2}}}
$$

where $\gamma=\sqrt{1+\Pi^{2}}$ is the Lorentz factor. Taking into 
account the relation between the electric and magnetic field strengths of a transverse e.m. wave in a medium with a refraction index $n$ (hereafter $n>1$ ),

$$
\xi_{H}\left(t^{\prime}, \mathbf{r}^{\prime}\right)=n\left[\nu_{0} \xi_{E}\left(t^{\prime}, \mathbf{r}^{\prime}\right)\right],
$$

Eq. (1) may be represented in the form

$$
\begin{aligned}
\frac{d \Pi}{d t^{\prime}}= & {\left[1-\frac{n}{\sqrt{1+\Pi^{2}}}\left(\nu_{0} \Pi\right)\right] \xi_{E}\left(t^{\prime}, \mathbf{r}^{\prime}\right) } \\
& +\frac{n}{\sqrt{1+\Pi^{2}}} \nu_{0}\left(\Pi \xi_{E}\left(t^{\prime}, \mathbf{r}^{\prime}\right)\right) .
\end{aligned}
$$

For a plane e.m. wave of an arbitrary form

$$
\xi_{E}\left(x^{\prime}, t^{\prime}\right)=\xi\left(n x^{\prime}-t^{\prime}\right)
$$

the integration of Eq. (5) with Eq. (4) has been made analytically in [6] and the nonlinear threshold phenomenon of charged particle reflection or capture by a plane transverse e.m. wave in a dielectriclike medium revealed. However, the analytical treatment of the entire dynamics of a particle law of motion corresponding to the mentioned phenomenon is impossible even for a plane wave. Consequently, we will treat this problem and then particle acceleration by actual laser pulses via numerical integration of equations of the particle law of motion $\mathbf{r}^{\prime}=\mathbf{r}^{\prime}\left(t^{\prime}\right)$, i.e., Eq. (2) with Eq. (5).

For concreteness, we will assume that a plane e.m. wave pulse has a linear polarization:

$$
\xi_{E x}=\xi_{E z}=0, \quad \xi_{E y}\left(x^{\prime}, t^{\prime}\right)=\xi\left(n x^{\prime}-t^{\prime}\right) .
$$

Then Eq. (5) can be written via projections in the following form:

$$
\begin{aligned}
\frac{d \Pi_{x}}{d t^{\prime}} & =\frac{n}{\sqrt{1+\Pi^{2}}} \Pi_{y} \xi\left(n x^{\prime}-t^{\prime}\right), \\
\frac{d \Pi_{y}}{d t^{\prime}} & =\left(1-\frac{n}{\sqrt{1+\Pi^{2}}} \Pi_{x}\right) \xi\left(n x^{\prime}-t^{\prime}\right), \\
\frac{d \Pi_{z}}{d t^{\prime}} & =0 .
\end{aligned}
$$

From the first equation of (6) and Eq. (4) follows the integral of motion

$$
K=\gamma\left(t^{\prime}\right)-\frac{1}{n} \Pi_{x}\left(t^{\prime}\right)=\text { const }
$$

which allows us, after the integration of the equations for transversal motion, to find the particle energy in the field:

$$
\begin{aligned}
\gamma\left(t^{\prime}\right)= & \frac{\gamma_{0}}{n^{2}-1}\left\{n ^ { 2 } ( 1 - \frac { \beta _ { 0 x } } { n } ) \mp \left[\left(1-n \beta_{0 x}\right)^{2}\right.\right. \\
& \left.\left.-\frac{n^{2}-1}{\gamma_{0}^{2}}\left\{\xi^{2}\left[x^{\prime}\left(t^{\prime}\right), t^{\prime}\right]-2 \Pi_{0 y} \xi\left[x^{\prime}\left(t^{\prime}\right), t^{\prime}\right]\right\}\right]^{1 / 2}\right\} .
\end{aligned}
$$

Here $\beta_{0 x}=\mathrm{v}_{0 x} / c$ and $\mathrm{v}_{0 x}, \gamma_{0}, \Pi_{0 y}$ are the initial values of longitudinal velocity, normalized energy, and transversal momentum of the particle, respectively. According to Eq. (8) there is a critical value of the wave intensity in a medium with a refraction index $n>1$ above which (when the expression under the root becomes zero) the particlewave interaction dynamics results in the above-mentioned phenomenon of the particle reflection from the wave pulse [6]. To illustrate this peculiarity of nonlinear interaction of a charged particle with a strong e.m. wave in a medium with the effect of acceleration, we will present the graphics of numerical solutions of Eq. (6) for the laser pulse of finite duration, neglecting at first the transversal profile of an actual laser beam that corresponds to a plane wave in Eq. (8).

For the numerical integration of Eqs. (2) and (6), we will choose a definite form for a plane e.m. wave pulse:

$$
\xi(\tau)=\frac{\xi_{0}}{\cosh \left(\frac{\tau}{\tau_{0}}\right)} \cos \tau
$$

where $\tau=n x^{\prime}-t^{\prime}$ is the wave coordinate, $\tau_{0}$ is the pulse duration, and $\xi_{0}$ is the peak value of the wave-pulse intensity parameter.

The particle initial energy is taken to be $\mathcal{E}_{0}=40 \mathrm{MeV}$ and the initial velocity is directed at the angle $\vartheta=9 \times$ $10^{-3}$ rad to the wave propagation direction $\left(p_{0 z}=0\right)$. The refractive index of the gaseous medium for this calculation has been chosen to be $n_{0}-1=10^{-4}$. Figure 1 illustrates the evolution of the particle energy: the energy versus the position $x$ is plotted for a neodymium laser $\left(\hbar \omega_{0} \simeq\right.$ $1.17 \mathrm{eV}$ ) with electric field strength $E_{0}=3 \times 10^{8} \mathrm{~V} / \mathrm{cm}$ and $\tau_{0}=8 \pi$. For these parameter values, the wave intensity is above the critical point and, as we see from this figure, the particle energy is abruptly changed corresponding to the reflection phenomenon. Figure 2(a) illustrates the evolution of the energies of particles with different initial interaction angles. The initial energies for all particles are $\mathcal{E}_{0} \simeq 40 \mathrm{MeV}$. Figure 2(b) illustrates the role of initial

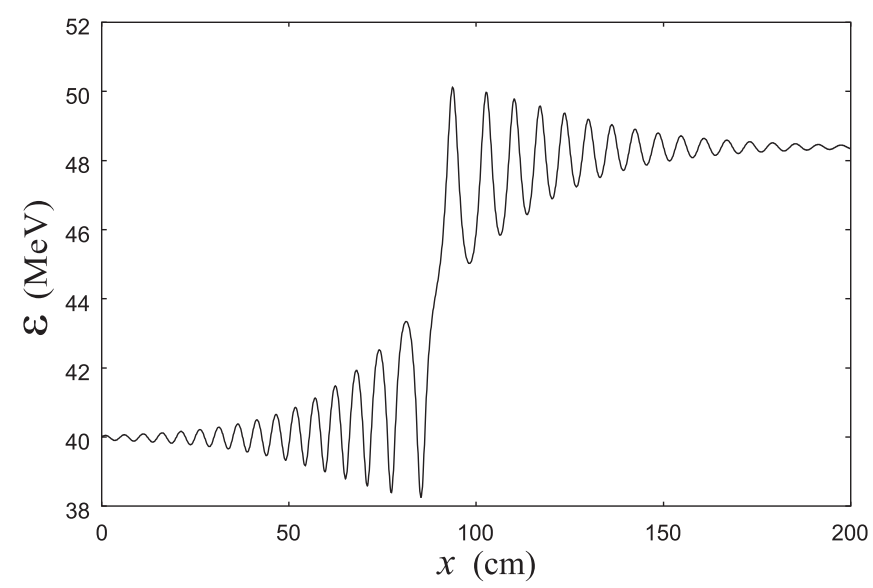

FIG. 1. Reflection of the particle. The energy versus the position $x$ is plotted when the wave intensity is above the critical point. 

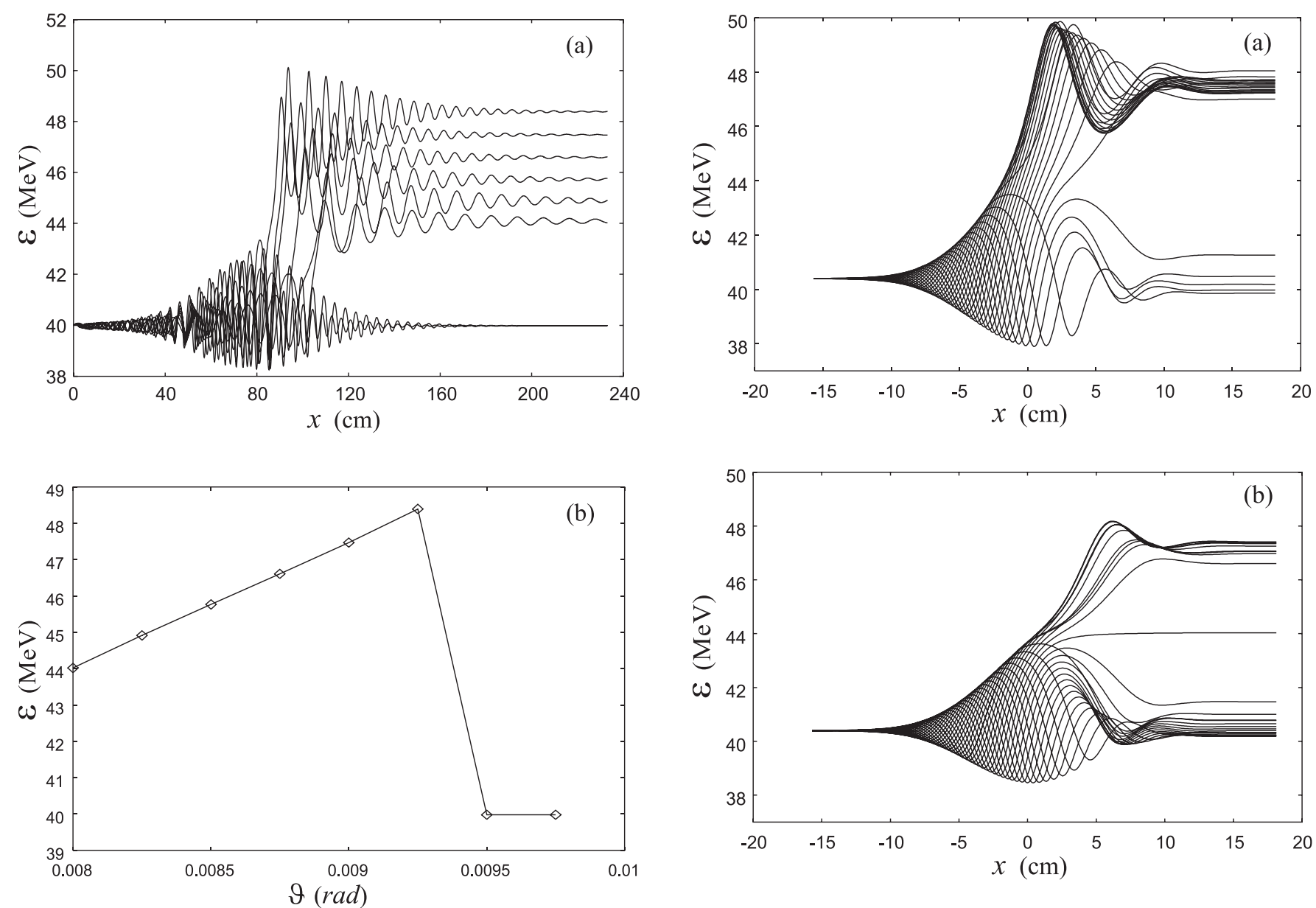

FIG. 2. Reflection of the particles with different initial interaction angles. Panel (a) displays the evolution of the energies of particles. In (b) the final energy versus the interaction angle is plotted.

conditions: the final energy versus the interaction angle is plotted. As follows from Eq. (8), the critical intensity as well as the final energy depend on the initial interaction angle and as a consequence we have this picture. Note that the acceleration rate neither depends on the field magnitude (only should be above-threshold field) nor on the interaction length.

To demonstrate the dependence of the considered process on a transversal profile of the laser intensity for actual beams in Fig. 3, the evolution of the energies of particles with various initial phases (with initial energies $\mathcal{E}_{0} \simeq$ $40 \mathrm{MeV}$ ) is illustrated. The laser beam transversal profile is modeled by the Gaussian function

$$
\xi\left(y^{\prime}, z^{\prime}, \tau\right)=\xi_{0} \exp \left(-\frac{4}{d^{\prime 2}}\left(y^{\prime 2}+z^{\prime 2}\right)\right) \frac{\cos \tau}{\cosh \left(\frac{\tau}{\tau_{0}}\right)}
$$

with the normalized transverse width $d^{\prime}=2 \pi \times 10^{3}, \tau_{0}=$ $100 \pi$. As we see from this figure the acceleration picture is essentially changed depending on the entrance coordinates

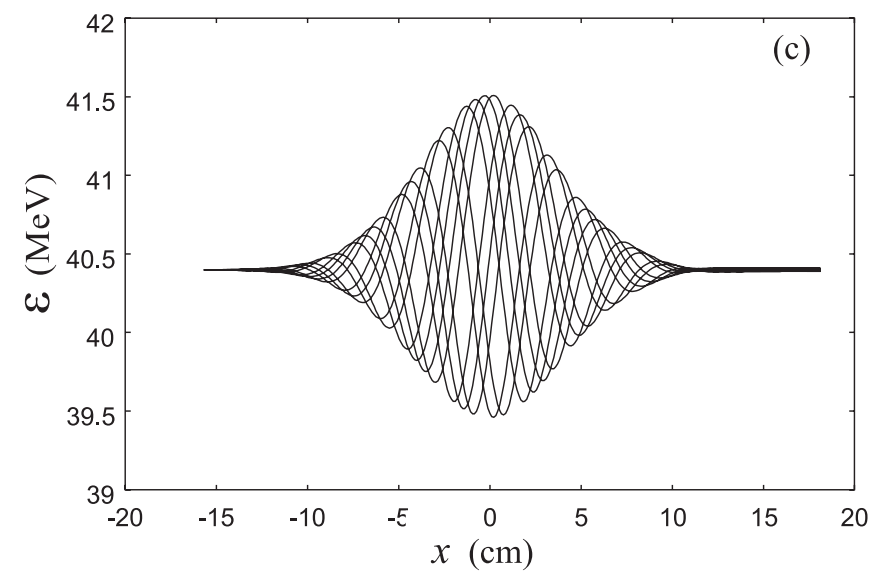

FIG. 3. The evolution of the energies of particles with various initial phases are shown for the laser beam with transversal intensity profile for the various entrance coordinates: (a) $z=$ 0 , (b) $z=d / 4$, and (c) $z=d / 2$.

of the particles. This is the manifestation of the threshold nature of the reflection phenomenon.

To illustrate the evolution of particle reflection from the laser pulse in Fig. 4, we present the graphics of numerical simulations for the distance between the particles and laser beam center in the whole interaction process for different 


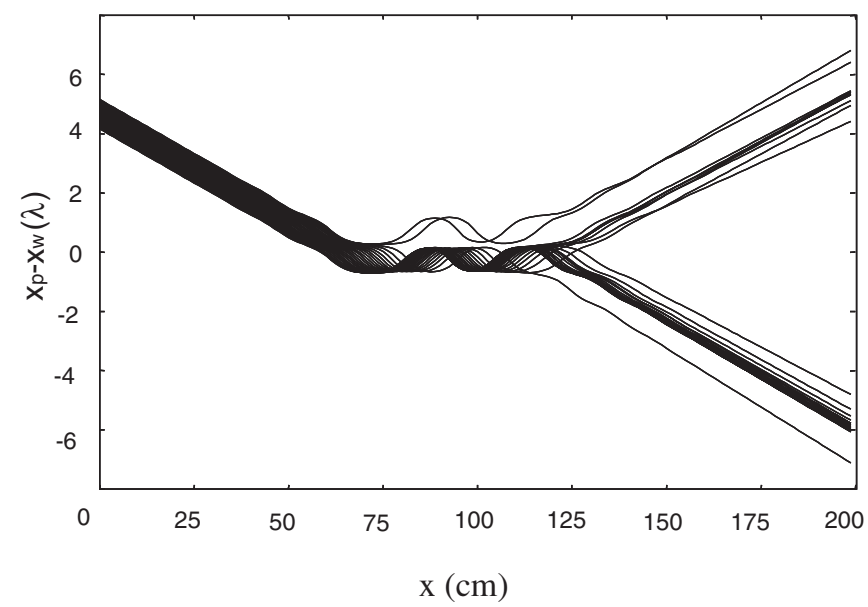

FIG. 4. Evolution of particles reflection from laser pulse via the distance between the particles and pulse center (for a wave pulse with the normalized transverse width $d^{\prime}=\pi \times 10^{4}$ ).

initial angles between the particle-wave propagation directions. As is seen from this graphics, there is a distance of simultaneous motion of the particles with the wave, which corresponds to nonlinear Cherenkov resonance in the field at the critical value of the wave intensity [see Eq. (8)]. The effective acceleration occurs on this distance as a coherent accumulation of inverse Cherenkov effect (induced absorption of the wave photons). In opposite initial conditions if the particles overtake the wave - the coherent accumulation of induced Cherenkov radiation occurs on this distance and in the result the effective deceleration of the particles takes place. So, in both cases after the coherent accumulation of induced Cherenkov effect on this distance the particles leave the wave-reflection (see the graphics for inverse Cherenkov effect when the acceleration takes place).

\section{PARTICLE ACCELERATION IN THE CAPTURE REGIME}

Let us now consider the particle acceleration due to the capture phenomenon [29,32]. In the capture regime with the constant refraction index of a medium, the longitudinal velocity of the particle oscillates in time remaining at the average constant and net energy change is zero. However, if one changes the refraction index of a medium along the wave propagation direction, continuously increasing the phase velocity of the wave, it is possible to accelerate a particle in the capture regime [32]. This is just the case of large acceleration. The variation law of the medium refractive index in this regime should be defined in a selfconsistent manner (continuous curve in Fig. 5).

To illustrate the particle acceleration in the capture regime, we will represent the results of numerical solution of Eqs. (1) and (2) in the field of an actual laser beam with the electric field strength,

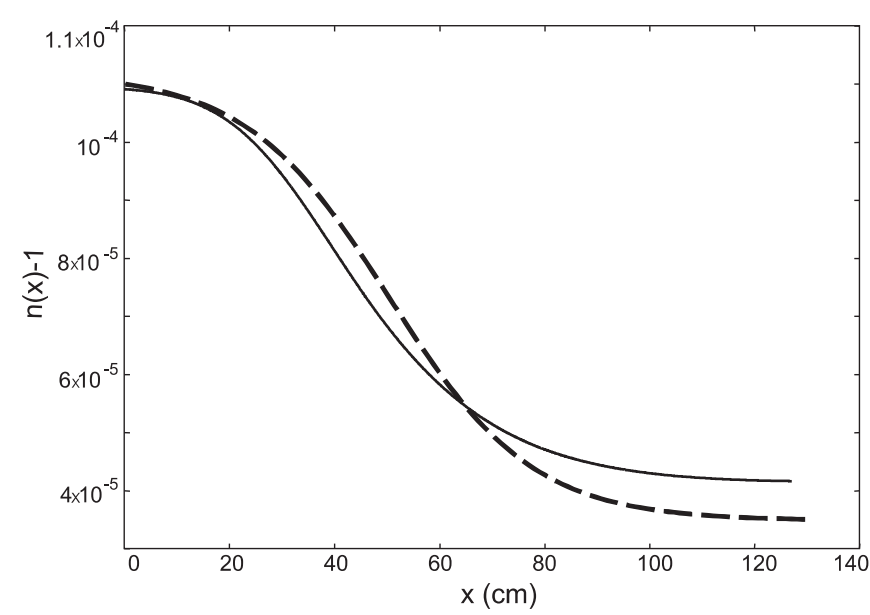

FIG. 5. Variation of refractive index of a medium. The continuous curve represents a self-consistent variation law; the dashed-line curve corresponds to an approximated one by Eq. (12).

$$
\begin{aligned}
\xi_{E y}\left(y^{\prime}, z^{\prime}, \tau\right)= & \xi_{0} \exp \left(-\frac{4}{d^{\prime 2}}\left(y^{\prime 2}+z^{\prime 2}\right)\right) \\
& \times \frac{\cos \left[\int n\left(x^{\prime}\right) d x^{\prime}-t^{\prime}+\varphi_{0}\right]}{\cosh \left(\frac{\int n\left(x^{\prime}\right) d x^{\prime}-t^{\prime}+\varphi_{0}}{\tau_{0}}\right)}, \\
\xi_{E x}= & 0, \quad \xi_{E z}=0,
\end{aligned}
$$

where $\varphi_{0}$ is the wave phase corresponding to the particle initial state.

Simulations have been made for neodymium laser $\left(\hbar \omega_{0} \simeq 1.17 \mathrm{eV}\right)$ with electric field strength $E_{0}=$ $3 \times 10^{8} \mathrm{~V} / \mathrm{cm}$ and $\tau_{0}=2 \pi \times 10^{3}, d^{\prime}=10^{4} \pi$. The analysis of numerical investigation shows that the self-consistent variation law for the refractive index of the medium may be approximated by the function

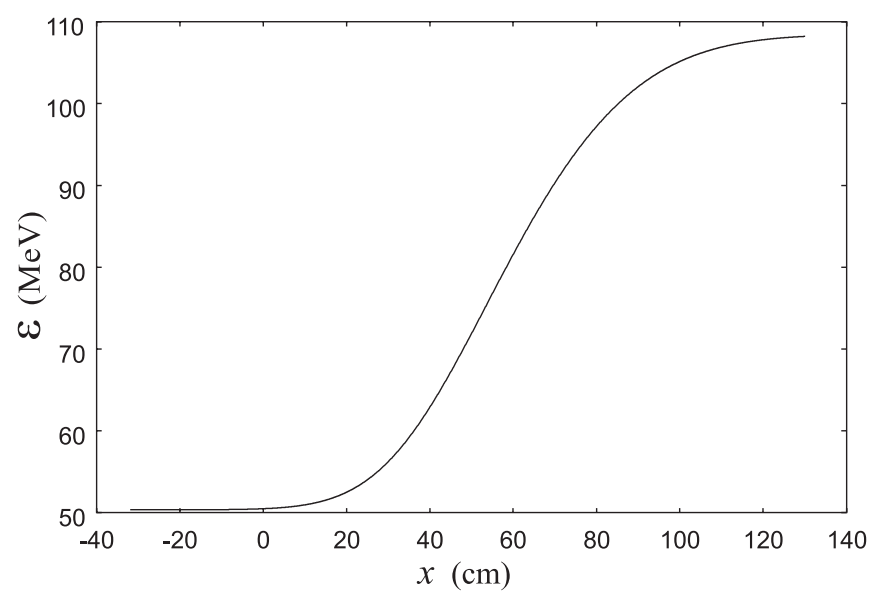

FIG. 6. The evolution of the particle energy in the capture regime with variable refractive index. 

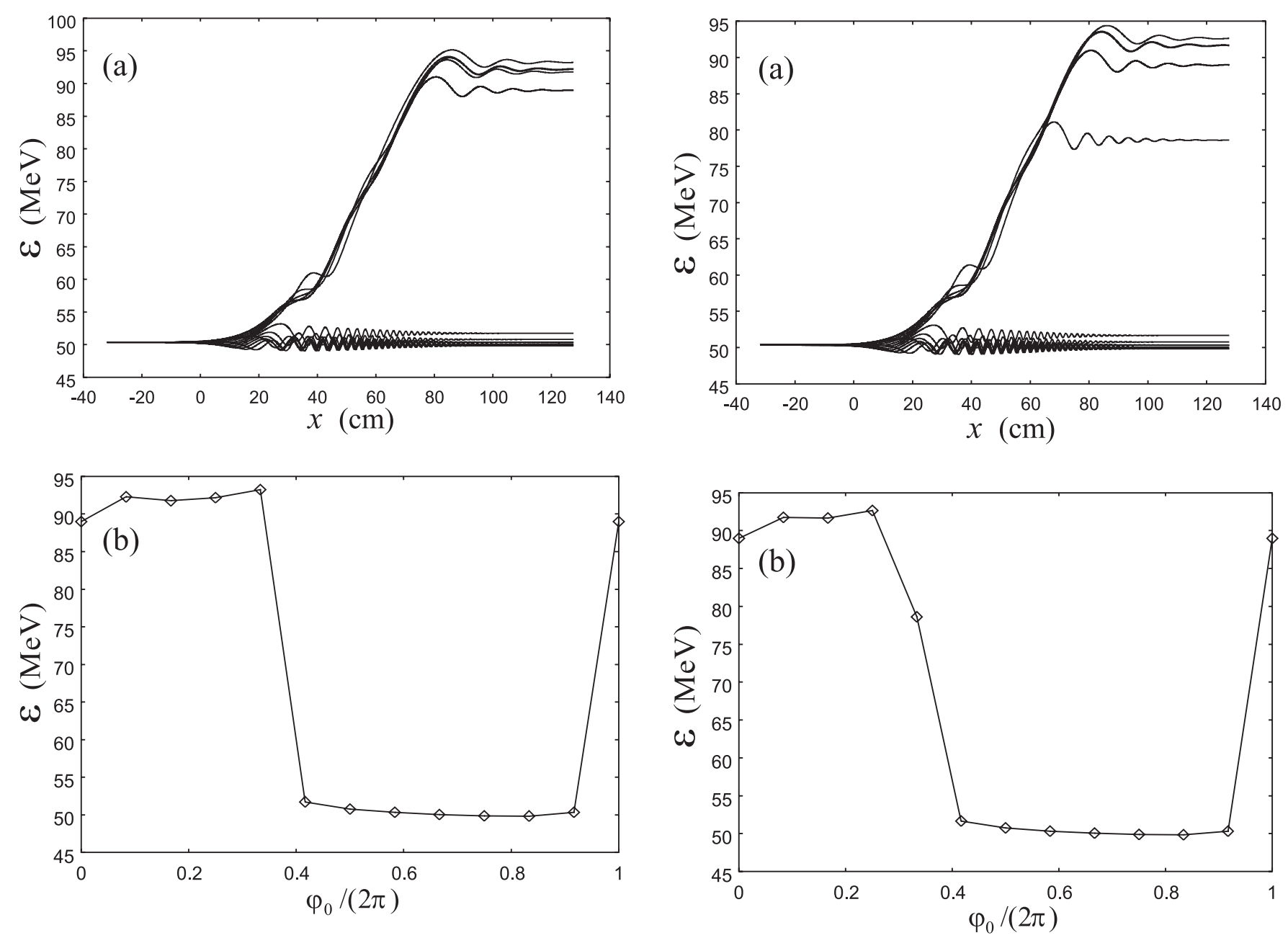

FIG. 7. Acceleration of the particles in the capture regime. Panel (a) displays the evolution of the energies of particles with various initial phases. The initial entrance coordinate is $z=0$. In (b) the final energy versus the initial phase is plotted.

$$
n(x)=\frac{n_{0}+n_{f}}{2}+\frac{\left(n_{f}-n_{0}\right)}{2} \tanh (\kappa x),
$$

where $n_{0}, n_{f}$ are the initial and final values of the refractive index of a gaseous medium and $\kappa$ characterizes its decreasing rate (dashed-line curve in Fig. 5).

Figure 6 illustrates the evolution of the particle energy in the capture regime. The particle initial energy is taken to be $\mathcal{E}_{0}=50.5 \mathrm{MeV}$ and the initial velocity is directed at the angle $\vartheta=9 \times 10^{-3}$ rad to the wave propagation direction $\left(p_{0 z}=0\right)$. The initial value of the medium refractive index has been chosen to be $n_{0}-1 \simeq 10^{-4}$. As we see in the capture regime with variable refractive index, one can achieve considerable acceleration.

To show the role of initial conditions in Fig. 7(a), the evolution of the energies of particles with the same initial energies $\mathcal{E}_{0}=50.5 \mathrm{MeV}\left(\vartheta=9 \times 10^{-3} \mathrm{rad}\right)$ and various initial phases is illustrated. The initial entrance coordinate is $z=0$. Figure 7(b) displays the role of initial conditions: the final energy versus the initial phase is plotted. In Fig. 8

FIG. 8. Acceleration of the particles in the capture regime. Panel (a) displays the evolution of the energies of particles with various initial phases. The initial entrance coordinate is $z=$ $0.25 \mathrm{~mm}$. In (b) the final energy versus the initial phase is plotted.

the parameters are the same as in Fig. 7(a) except the initial entrance coordinate, which is taken to be $z=0.25 \mathrm{~mm}$. As we see from these figures the captured particles are accelerated, while the particles situated in the unstable phases (or if the conditions for capture are not fulfilled) after the interaction remain with the initial energy.

\section{CONCLUSION}

We have presented a theoretical and numerical treatment of the charged particle nonlinear dynamics in the field of a strong electromagnetic wave pulse of finite duration and certain form of the envelope. The acceleration due to the nonlinear threshold phenomena of a particle reflection and capture by actual laser pulses in dielectric-gaseous media has been shown. These phenomena, which have been revealed previously for a plane electromagnetic wave and result from the induced nonlinear Cherenkov effect, have 
been developed for the wave pulses of finite duration and transverse-space envelopes. The obtained numerical results prove the particle reflection and capture phenomena in the field of actual laser pulses with temporal and space profiles and, on the other hand, lead to the particle acceleration. In the reflection regime, particle acceleration takes place already at the constant refraction index of a medium, meanwhile in the capture regime it is necessary to decrease adiabatically the refraction index of a gaseous medium along the pulse propagation direction by the certain selfconsistent variation law corresponding to acceleration rate.

\section{ACKNOWLEDGMENTS}

This work was supported by International Science and Technology Center (ISTC) Project No. A-1307.

[1] A. Ogata and K. Nakajima, Laser Part. Beams 16, 381 (1998).

[2] S. P. D. Mangles et al., Phys. Rev. Lett. 94, 245001 (2005).

[3] S. P. D. Mangles et al., Phys. Rev. Lett. 96, 215001 (2006).

[4] R. Wagner, S.-Y. Chen, A. Maksimchuk, and D. Umstadter, Phys. Rev. Lett. 78, 3125 (1997).

[5] T. Tajima and J. M. Dawson, Phys. Rev. Lett. 43, 267 (1979).

[6] E. Esarey et al., IEEE Trans. Plasma Sci. 24, 252 (1996); IEEE J. Quantum Electron. 33, 1879 (1997).

[7] Th. Stohlker et al., Phys. Rev. Lett. 86, 983 (2001).

[8] M. Drescher et al., Science 291, 1923 (2001).

[9] A. Pukhov and J. Meyer-ter-Vehn, Appl. Phys. B 74, 355 (2002).

[10] S. V. Tochitsky et al., Phys. Rev. Lett. 92, 095004 (2004).

[11] S. P. D. Mangles et al., Nature (London) 431, 535 (2004).

[12] C. G. R. Geddes et al., Nature (London) 431, 538 (2004).

[13] J. Faure et al., Nature (London) 431, 541 (2004).

[14] S. Fritzler et al., Phys. Rev. Lett. 92, 165006 (2004).

[15] E. Miura et al., Appl. Phys. Lett. 86, 251501 (2005).
[16] B. Hidding et al., Phys. Rev. Lett. 96, 105004 (2006).

[17] M. I. K. Santala et al., Phys. Rev. Lett. 86, 1227 (2001); Science 298, 1596 (2002).

[18] K. Nakajima et al., Phys. Rev. Lett. 74, 4428 (1995).

[19] P. Mora and B. Quesnel, Phys. Rev. Lett. 80, 1351 (1998).

[20] K. T. McDonald, Phys. Rev. Lett. 80, 1350 (1998).

[21] K. Nakajima, Laser Part. Beams 18, 519 (2000).

[22] P. X. Wang et al., Appl. Phys. Lett. 78, 2253 (2001).

[23] Y. I. Salamin and C. H. Keitel, Phys. Rev. Lett. 88, 095005 (2002).

[24] Ch. Varin, M. Piche, and M. A. Porras, Phys. Rev. E 71, 026603 (2005).

[25] K. Shimoda, Appl. Opt. 1, 33 (1962).

[26] J. R. Fontana and R. H. Pantell, J. Appl. Phys. 53, 5435 (1982).

[27] J. A. Edighoffer, W. D. Kimura, R. H. Pantell, M. A. Piestrup, and D. Y. Wang, Phys. Rev. A 23, 1848 (1981).

[28] W. D. Kimura et al., Phys. Rev. Lett. 74, 546 (1995).

[29] V. M. Haroutunian and H. K. Avetissian, Sov. J. Quantum Electron. 2, 39 (1972).

[30] V. M. Haroutunian and H. K. Avetissian, Phys. Lett. 44A, 281 (1973).

[31] V. M. Haroutunian and H. K. Avetissian, Zh. Eksp. Teor. Fiz. 62, 1639 (1972) [Sov. Phys. JETP 35, 854 (1972)].

[32] H. K. Avetissian, Relativistic Nonlinear Electrodynamics (Springer-Verlag, New York, 2006).

[33] V. M. Haroutunian and H. K. Avetissian, Phys. Lett. 59A, 115 (1976).

[34] H. K. Avetissian et al., Phys. Lett. 66A, 161 (1978).

[35] H. K. Avetissian and S. G. Oganesyan, Sov. J. Izv. Acad. Nauk Arm. SSR, Fiz. 8, 12 (1973).

[36] H. K. Avetissian, Usp. Fiz. Nauk 167, 793 (1997) [Phys. Usp. 40, 755 (1997)].

[37] C. J. McKinstrie and E. A. Startsev, Phys. Rev. E 54, R1070 (1996); Phys. Rev. E 56, 2130 (1997).

[38] K. Nakajima, Laser Phys. 13, 421 (2003).

[39] I. Smetanin and K. Nakajima, Laser Phys. 13, 1 (2003). 